\title{
Effect of Baduanjin Sequential Therapy on the Quality of Life and Cardiac Function in Patients with AMI After PCI: A Randomized Controlled Trial
}

\author{
Ming-Gui Chen, Xuefei Liang, Lili Kong, Jingjing Wang, Fangfang Wang, Xiyan Hu, \\ Jianzhuo He, Rui-Xiang Zeng, Shuai Mao, Liheng Guo, Min-Zhou Zhang $\mathbb{D}$, \\ and Xiaoxuan Zhang \\ Division of Chest Pain Center, The Second Affiliated Hospital of Guangzhou University of Chinese Medicine, \\ The Second Clinical College of Guangzhou University of Chinese Medicine, Guangzhou 510120, China
}

Correspondence should be addressed to Min-Zhou Zhang; minzhouzhang@aliyun.com and Xiaoxuan Zhang; zhxiaoxuan2003@ 163.com

Received 21 March 2020; Accepted 2 June 2020; Published 4 July 2020

Academic Editor: Avni Sali

Copyright (C) 2020 Ming-Gui Chen et al. This is an open access article distributed under the Creative Commons Attribution License, which permits unrestricted use, distribution, and reproduction in any medium, provided the original work is properly cited.

Purpose. The purpose of this study was to examine the effects of Baduanjin sequential therapy (BST) on the quality of life and cardiac function in patients with AMI after PCI. Subjects. 96 patients with AMI after PCI were randomly assigned as subjects to two groups: BST group who received 24 weeks of BST training and control group who received no training. Methods. The methods used in this study included the changes in SF-36 subscales, the measures of left ventricular ejection fraction (LVEF), N-terminal pro-B-type natriuretic peptide (NT-pro-BNP), the body mass index (BMI), and the abdominal circumference. Results. Of the 96 participants, 82 total patients completed the entire study. At 12 weeks, role physical and health transition of SF-36 were significantly different between the two groups, with a difference of 26.12 (95\% CI, 11.59 to 40.64$)$ in role physical and a difference of 15.94 (95\% CI, 5.60 to 26.28$)$ in health transition $(p<0.05)$. However, there were statistically significant differences in all aspects of SF-36 between the two groups at 24 weeks $(p<0.05)$. The BST also lowered abdominal circumference and BMI as compared with the control group. In the 24-week follow-up, a significant difference was found in the decline of the LVEF in the control group $(p=0.020)$, while there was a nonsignificant difference in the BST group $(p=0.552)$. Compared with the control group, the BST group reduced $50 \mathrm{pg} / \mathrm{ml}$ on the NT-pro-BNP at 24 weeks $(p=0.013)$. The effects of BST exercise were maintained at 24 weeks after the intervention. No serious adverse events were observed. Conclusions. The BST appears to improve the quality of life in patients with AMI after PCI, with additional benefits of lowered abdominal circumference and BMI and improved level of cardiac function. This trial is registered with NCT02693795.

\section{Introduction}

Acute myocardial infarction (AMI) is one of the leading causes of death around the world, with the potential for substantial morbidity and mortality [1,2]. From 2001 to 2011, the number of AMI in China increased by four times [3]. Based on the estimation of the Markov model, 21 million new acute coronary events and 7 million cardiac deaths will occur in China in the next 20 years [4]. Although technical advances in percutaneous coronary intervention (PCI) are becoming better and more effective medical therapy, most patients still suffer from angina pectoris, anxiety, depression, and reduced cardiac function after PCI [5-8]. At least 5-10\% of survivors die within the first 12 months after the MI and close to $50 \%$ need hospitalization within the same year $[9,10]$.

Previous studies have shown that myocardial ischemiareperfusion (IR) injury can cause ventricular cell death and is a major pathological event leading to morbidity and mortality in those with acute myocardial infarction $[11,12]$. 
Interestingly, appropriate exercise can rapidly produce a cardiac phenotype that resists IR-induced myocardial injury. It can also relax the heart blood vessels, promote the regeneration of microvessel, and regulate the balance of the autonomic nervous system of the heart [13-15]. As a result, many studies have confirmed that exercise can reduce cardiovascular diseases and significantly decrease the incidence of angina and cardiac events [16, 17]. Exercise also improves patient survival and improves patients' quality of life $[18,19]$. However, most exercise programs are complicated and require supervision, and they may even result in an overload of the heart in patients with AMI. A simple easyto-learn, free-of-charge, and appropriate home-based exercise program is necessary to improve adherence to exercise and help patients with AMI maintain a physically active lifestyle.

Baduanjin exercise, as an important component of Chinese Qigong exercises, is easy to learn and can be performed at home without any assistive equipment [20]. Previous studies have found that Baduanjin exercise is beneficial to health, leading to improvement of strength, physical fitness, and depression [21-23]. However, many studies have focused on the effects of standing Baduanjin on chronic diseases. Baduanjin sequential therapy (BST) which has been developed by our team consists of sitting and standing forms as shown in Supplementary Appendix Figures 1(a) and 1(b). It is simple and effective in clinical practice and the program emphasizes early rehabilitation interventions and continued rehabilitation after discharge. However, there exists few data from large-scale randomized trials that have addressed the effect of Baduanjin exercise on the quality of life and cardiac function in the patient with AMI after PCI. The primary aim of this study was to examine the quality of life and the level of cardiac function in AMI patients after PCI over time in response to a 24-week BST.

\section{Materials and Methods}

2.1. Research Design. This study was a parallel, randomized controlled trial (RCT) with a longitudinal research design. Participants were randomly assigned to either the BST group or the control group. Our study strictly complies with the guidelines of the CONSORT 2010 checklist. The study received ethical approval from the Guangdong Provincial Hospital of Chinese Medicine's Human Research Ethics Committee (approval number B2015-41-01), and it was registered with the clinicaltrials.gov (protocol: NCT02693795). This study complies with the principles outlined in the Helsinki Declaration and the relevant regulations of the national regulatory agency.

2.2. Participants and Procedures. Recruitment took place in 2016 and 2017, primarily via the screening of hospitalized patients. Eligibility criteria included patients with a clinical diagnosis of AMI, aged 18 to 80 years, performed PCI, and signed an informed consent form. The AMI diagnostic criteria are a reference to the third universal definition of myocardial infarction [24]. Exclusion criteria were current participation in any other behavioral or pharmacological study or instructor-led exercise program, cardiogenic shock, severe heart failure (NYHA cardiac function class IV or LVEF $\leq 35 \%$ ), malignant arrhythmia (ventricular fibrillation, ventricular tachycardia, and frequent ventricular premature beats), or active bleeding. Patients with poor underlying conditions such as those suffering from bone and joint disease or nervous system disorders that would impede full participation in the study and unavailability during the study period were also excluded.

Patients were randomized to either the BST group or to the control group in a $1: 1$ allocation ratio. Biostatisticians randomized assignments using a sequence randomly generated in the $\mathrm{R}$ statistical package. These assignments were put into sealed, opaque envelopes with the date and signature labels placed over the seals of the envelopes. After baseline evaluation of patients, the study coordinator opened the consecutive randomization envelopes and informed the individuals of their group assignments.

Given the nature of the exercise intervention, it was impossible that study participants and exercise instructors could be unaware of the randomization allocation throughout the entire duration of this trial. However, the investigators conducting the outcome assessments and the biostatisticians responsible for the analysis were blinded to the assignment.

The patients in the BST group were asked to complete attendance forms as well as class sign-in sheets for the BST intervention once a month and the patients in the control group would be requested to maintain their original habit of lifestyle. They were also asked to maintain Baduanjin exercise or regular aerobic exercise throughout the follow-up period. Adherence was measured after intervention by telephone calls or WeChat during which research assistants inquired about the frequency and duration of the patients' participation in the BST exercise and general aerobic exercise.

2.3. Sample Size. The sample size was calculated on the basis of the changes in SF-36 after 24 weeks of intervention between comparison groups with a significance level of $5 \%$ and a two-tailed critical region. According to previous research, the control group can increase the average score of SF-36 by 9 points. Baduanjin can increase the average score of SF-36 by 23 points, and the standard deviation is about 29 . According to the formula, $N=[(Z 1-\alpha+Z 1-\beta)(s / \Delta)]^{2}$. The sample size of each group is about 40 cases and a total of 80 cases in the two groups. Considering the situation of dropout and loss of follow-up, it is estimated that the dropout (loss of follow-up) of $20 \%$ will require 96 cases.

\subsection{Interventions}

2.4.1. BST Exercise. BST has been developed by our team based on the characteristics of patients with myocardial infarction (Supplementary Appendix Video 1). It consists of the sitting form and the standing form (Supplementary Appendix Figures 1(a) and 1(b)) because the goal is to allow patients with AMI to be also rehabilitated during 
hospitalization. On the second day after the standard treatment (blood revascularization + drug therapy), patients began the sitting Baduanjin exercises $(30 \mathrm{~min} / \mathrm{session}$, twice a day, 3 days). After discharge, the patients did the standing Baduanjin exercises ( $30 \mathrm{~min} / \mathrm{session}$, fifth weekly, lasting up to 24-weeks). Patients were provided with a picture-based educational brochure describing the Baduanjin exercises including practicing techniques and safety precautions. Scheduled telephone follow-ups were also provided to reinforce patients' adherence to the 24-week follow-up assessments.

We had strict monitoring indicators and the therapy would be terminated if patients had any of the following conditions. (1) The patient felt laborious and had physical discomforts such as chest pain, dizziness, sweating, and severe dyspnoea. The oxygen saturation was $<90 \%$. (2) The $\mathrm{FIO}_{2}$ was $>60 \%$ and breathing was $>35$ breaths per minute. (3) The systolic blood pressure was $<90$ or $>200 \mathrm{mmHg}$ and mean arterial pressure $<65 \mathrm{mmHg}$. (4) The patient had unstable heart rhythm or needed to use antiarrhythmic drugs or vasoactive medicines.

2.5. Outcome Measures. All outcomes were measured at baseline (week 0) and midterm (week 12) and study completion (week 24). The primary outcome was the SF-36. The secondary outcomes included changes in LVEF, NT-proBNP, BMI, and abdominal circumference.

2.5.1. SF-36. The SF-36 health survey scale was developed by the health research institute of New England medical center in Boston, USA, and the Chinese version was successfully developed by the team of Li et al. in 2002, with good reliability and validity [25]. The SF-36 is a widely used, standard instrument for evaluating HRQoL. It is a self-assessment health status questionnaire containing 36 items (questions) about sociodemographic data, health, and personal behavior, grouped into 8 multi-item domains. It measures the following: (1) physical functioning (10 items), (2) social functioning (2 items), (3) role limitations because of physical problems (4 items), (4) role limitations because of emotional problems (3 items), (5) mental health (5 items), (6) energy and vitality (4 items), (7) bodily pain ( 2 items), and ( 8 ) general health perception (5 items). Each of the scores for the domains was coded, summed in an Excel chart, and later transformed in the statistical program SPSS. The results were presented from 0 (worst possible health) to 100 (best imaginable health).

2.5.2. LVEF. The LVEF was obtained through echocardiography. Participants were asked to undergo echocardiographic measurements on the first day of admission, at 12 and 24 weeks after discharge.

2.5.3. NT-pro-BNP. The NT-pro-BNP was determined by sampled blood. All participants were tested for the NT-proBNP on admission. The participants were also notified by telephone or WeChat to have blood tests at 12 and 24 weeks after discharge. The laboratory instrument we used was
ROCHE, the model was cobae e602, and the reagent batch number was 37537600 .

2.5.4. BMI. The BMI is the weight in kilograms divided by the square of the height in meters. Participants were instructed to remove their shoes for measuring their height and weight, and the staff used consistent methods with each patient measurement.

2.5.5. The Abdominal Circumference. The abdominal circumference was measured by tapeline. Patients were asked to stand up and naturally relaxed. The staff measured the abdominal circumference at the navel level 3 times. The results were averaged to derive a range for each measure.

2.6. Statistical Analysis. Data were reported as mean and standard deviation for continuous variables and as percentages for categorical variables. All analyses were undertaken in SPSS version 22 (IBM Corp). Independent $t$-tests and chisquare $\left(\chi^{2}\right)$ tests were used to examine the homogeneity of demographic and clinical information and the quality of life between the two groups at baseline. Values are presented as mean \pm SD and percentage (\%) in tables and median with the interquartile range in figures. Repeated measures analysis of variance (ANOVA) was used to test for change over time (i.e., baseline, 12 weeks, and 24 weeks) in the quality of life and NT-pro-BNP for the intervention and control groups. Group comparisons were made by the Mann-Whitney $U$ test for continuous variables and the chi-square test for categorical variables. A $p$ value of $<0.05$ was considered significant.

\section{Results}

3.1. Characteristics at Baseline between the Two Groups. During the study period from July 2016 to August 2017, a total of 110 patients with AMI who underwent PCI were recruited according to the inclusion criteria. Of these patients, 10 refused to participate, and 4 were unable to play videos at home. Most participants who refused to participate in this study did so for personal reasons, including being too busy, having no motivation or interest, and feeling inconvenienced or uncomfortable. 96 patients were randomly assigned to either the BST group $(n=48)$ or the control group $(n=48) .14$ patients withdrew due to personal reasons $(n=12)$, loss of contact $(n=1)$, and death $(n=1)$. Of the 96 participants, 82 total patients completed the entire study, yielding an attrition rate of $14.58 \%$ (Figure 1).

We obtained complete data from 82 cases as shown in Table 1. Table 1 shows the baseline characteristics of the study population. The groups were well matched about baseline characteristics, including age, sex, BMI, NT-proBNP, baseline study outcomes of drug treatment, and procedural characteristics $(p>0.05)$.

3.2. Primary Outcomes. Mean $( \pm \mathrm{SD})$ differences between groups in outcome visits at baseline, 12 weeks, and 24 weeks were shown in Table 2. Pairwise comparison and data analysis 


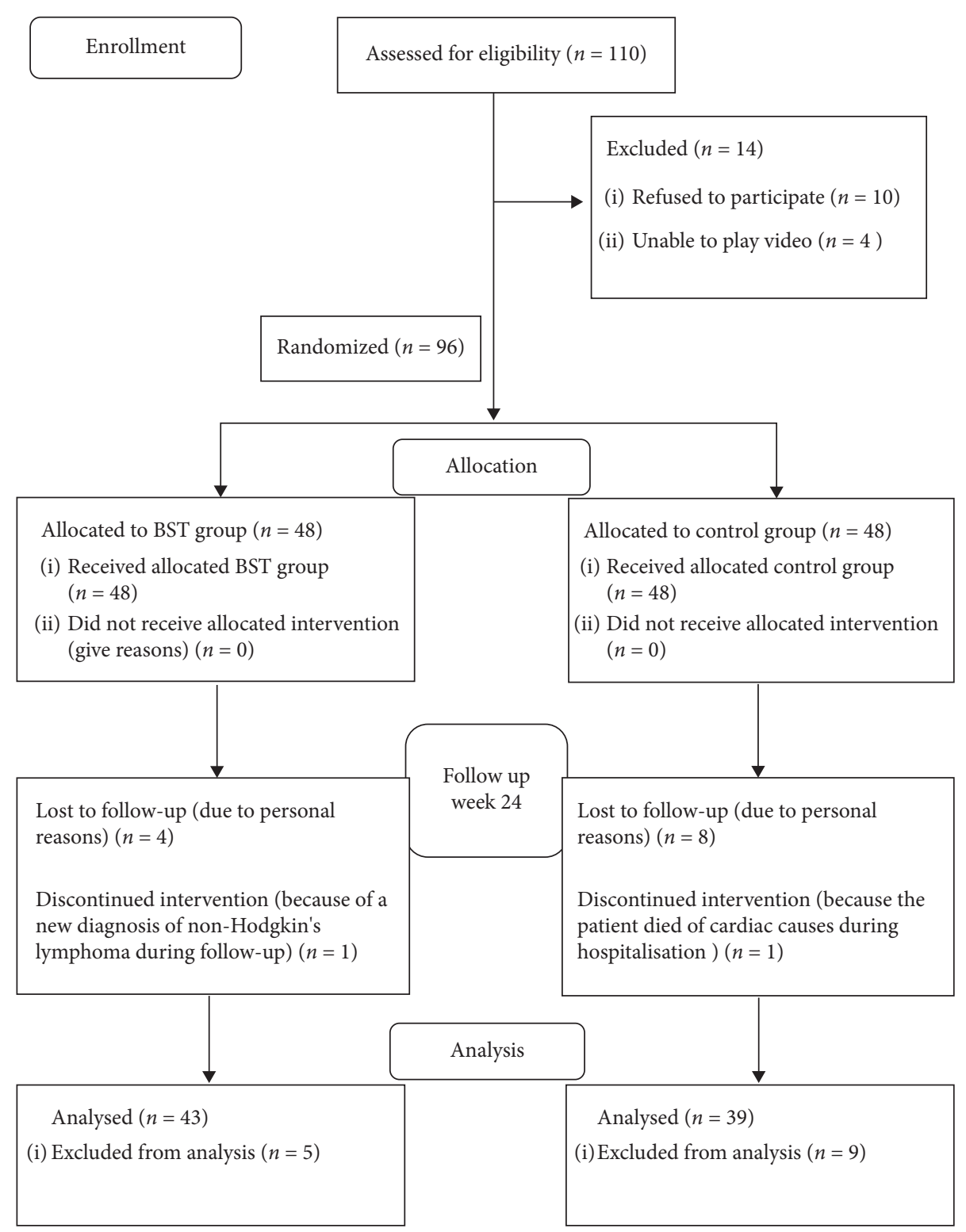

FIgURE 1: Inclusion and exclusion criteria for this study.

showed that there was no statistically significant difference between the two groups at the time of enrollment $(p>0.05)$. At 12 weeks, role physical and health transition of SF-36 were significantly different between the two groups, with a difference of 26.12 (95\% CI, 11.59 to 40.64$)$ in role physical and a difference of 15.94 (95\% CI, 5.60 to 26.28 ) in health transition $(p<0.05)$. However, there were statistically significant differences in physical functioning, role physical, bodily pain, general health, vitality, social function, role emotional, mental health, and health transition of SF-36 between the two groups at 24 weeks $(p<0.05)$.

3.3. Secondary Outcomes. Significant differences between groups were observed after 24 weeks. The BST group had significantly better performance on the abdominal circumference and BMI (Table 3). In the 12 weeks and 24 weeks, the participants in the BST group had a mean decrease of 3.45 centimeters and 4.29 centimeters in abdominal circumference compared with the control group $(p<0.05)$. Regarding BMI, although the 2 groups had no significant improvements during the 12 weeks, there was a considerable improvement in the BST group compared with the control group at 24 weeks $(23.40 \pm 2.30$ and $24.60 \pm 2.82$, respectively, $p=0.035)$.

In the 24-week follow-up, the LVEF of the BST group and the control group was baseline (61.06 \pm 9.64 and $63.74 \pm 8.51$, respectively), 12 -week outcome $(60.34 \pm 9.20$ and $60.48 \pm 9.64$, respectively), and 24-week outcome (58.74 \pm 10.10 and 57.79 \pm 9.56 , respectively) (Figure 2). A significant difference was found in the decline of the LVEF in the control group $(p<0.05)$. A nonsignificant difference was found in the decline of the LVEF in the BST group $(p>0.05)$. Besides, we also found that the BST group and the control group had a significant decrease in the NT-pro-BNP level at 
TABLE 1: Baseline clinical and procedural characteristics.

\begin{tabular}{|c|c|c|c|}
\hline Variable & Control group $(n=39)$ & BST group $(n=43)$ & $p$ value \\
\hline \multicolumn{4}{|l|}{ Clinical characteristics } \\
\hline Age (years) & $61.49 \pm 11.54$ & $59.98 \pm 10.86$ & 0.543 \\
\hline Male, $n(\%)$ & $30(76.9)$ & $29(67.4)$ & 0.34 \\
\hline Body mass index $\left(\mathrm{kg} / \mathrm{m}^{2}\right)$ & $24.62 \pm 2.86$ & $23.95 \pm 2.35$ & 0.25 \\
\hline Hypertension, $n(\%)$ & $22(56.4)$ & $26(60.5)$ & 0.71 \\
\hline Dyslipidaemia, $n(\%)$ & $11(28.2)$ & $14(32.6)$ & 0.669 \\
\hline History of smoker, $n(\%)$ & $25(64.1)$ & $22(51.2)$ & 0.237 \\
\hline History of CAD, $n(\%)$ & $1(2.6)$ & $6(14)$ & 0.148 \\
\hline Diabetes, $n(\%)$ & $10(25.6)$ & $9(20.9)$ & 0.614 \\
\hline $\operatorname{LVEF}(\%)$ & $63.74 \pm 8.52$ & $61.07 \pm 9.64$ & 0.189 \\
\hline $\operatorname{LVEDD}(\mathrm{mm})$ & $46.54 \pm 3.73$ & $44.83 \pm 4.68$ & 0.073 \\
\hline $\operatorname{LVESD}(\mathrm{mm})$ & $29.77 \pm 5.52$ & $29.82 \pm 5.71$ & 0.97 \\
\hline NT-pro-BNP (pg/ml) & $374(108-1093)$ & $408(191-921)$ & 0.66 \\
\hline WBC $\left(10^{9} / \mathrm{L}\right)$ & $10.55 \pm 3.12$ & $10.97 \pm 3.51$ & 0.574 \\
\hline $\mathrm{TG}(\mathrm{mmol} / \mathrm{L})$ & $1.51(1.09-2.19)$ & $1.53(1.10-2.30)$ & 0.795 \\
\hline $\mathrm{TC}(\mathrm{mmol} / \mathrm{L})$ & $4.86 \pm 0.85$ & $4.87 \pm 1.13$ & 0.971 \\
\hline HDL-C (mmol/L) & $1.10 \pm 0.28$ & $1.12 \pm 0.44$ & 0.856 \\
\hline LDL-C $(\mathrm{mmol} / \mathrm{L})$ & $3.38 \pm 0.78$ & $3.53 \pm 0.92$ & 0.425 \\
\hline \multicolumn{4}{|l|}{ Drug treatment } \\
\hline Statins, $n(\%)$ & $39(100)$ & $43(100)$ & \\
\hline Aspirin, $n(\%)$ & $39(100)$ & $43(100)$ & \\
\hline Clopidogrel, $n(\%)$ & $39(100)$ & $43(100)$ & \\
\hline b-Blockers, $n(\%)$ & $16(41)$ & $20(46.5)$ & 0.617 \\
\hline Calcium blockers, $n(\%)$ & $6(15.4)$ & $5(11.6)$ & 0.618 \\
\hline ACE inhibitors, $n(\%)$ & $6(15.4)$ & $3(7)$ & 0.388 \\
\hline ARBs, $n(\%)$ & $6(15.4)$ & $14(32.6)$ & 0.71 \\
\hline Diuretics, $n(\%)$ & $4(10.3)$ & $3(7)$ & 0.893 \\
\hline \multicolumn{4}{|l|}{ Procedural characteristics } \\
\hline Transradial access, $n(\%)$ & $36(92.3)$ & $39(90.7)$ & 1 \\
\hline \multicolumn{4}{|l|}{ Target vessel } \\
\hline Left main & $1(2.6)$ & $1(2.6)$ & 1 \\
\hline Left anterior descending & $32(82.1)$ & $33(76.7)$ & 0.554 \\
\hline Left circumflex & $20(51.3)$ & $19(44.2)$ & 0.521 \\
\hline Right coronary artery & $23(59)$ & $25(58.1)$ & 0.939 \\
\hline \multicolumn{4}{|l|}{ Lesion location } \\
\hline Proximal & $24(61.5)$ & $29(67.4)$ & 0.577 \\
\hline Middle & $27(69.2)$ & $27(62.8)$ & 0.539 \\
\hline Distal & $4(10.3)$ & $7(16.3)$ & 0.424 \\
\hline Branch & $13(33.3)$ & $13(30.2)$ & 0.763 \\
\hline Preoperative TIMI 0 & $17(43.6)$ & $18(41.9)$ & 0.874 \\
\hline Preoperative TIMI 1 & $2(5.1)$ & $1(2.3)$ & 0.931 \\
\hline Preoperative TIMI 2 & $1(2.6)$ & $2(4.7)$ & 1 \\
\hline Preoperative TIMI 3 & $19(48.7)$ & $22(51.2)$ & 0.825 \\
\hline Postoperative TIMI 3 & $39(100)$ & $43(100)$ & \\
\hline \multicolumn{4}{|l|}{ Lesion classification } \\
\hline Number of stents implanted & $1.18 \pm 0.389$ & $1.09 \pm 0.294$ & 0.263 \\
\hline
\end{tabular}

the 24-week outcome (105 [67-244], 155 [108-293], respectively, $p=0.013$ ) (Figure 3 );

3.4. Adverse Events. During the study period, 1 patient died of cardiac arrest during hospitalization in the control group, and 1 patient withdrew from the study because of a new diagnosis of non-Hodgkin"s lymphoma during follow-up in the
BST group. None of these events could be related to the interventions. 4 patients withdrew from the BST group, and 8 patients were removed from the control group for personal reasons.

\section{Discussion}

The results of this study showed that the 24-week BST program was effective for improving the quality of life and 
TABle 2: The changes of SF-36 subscales during the 24 weeks.

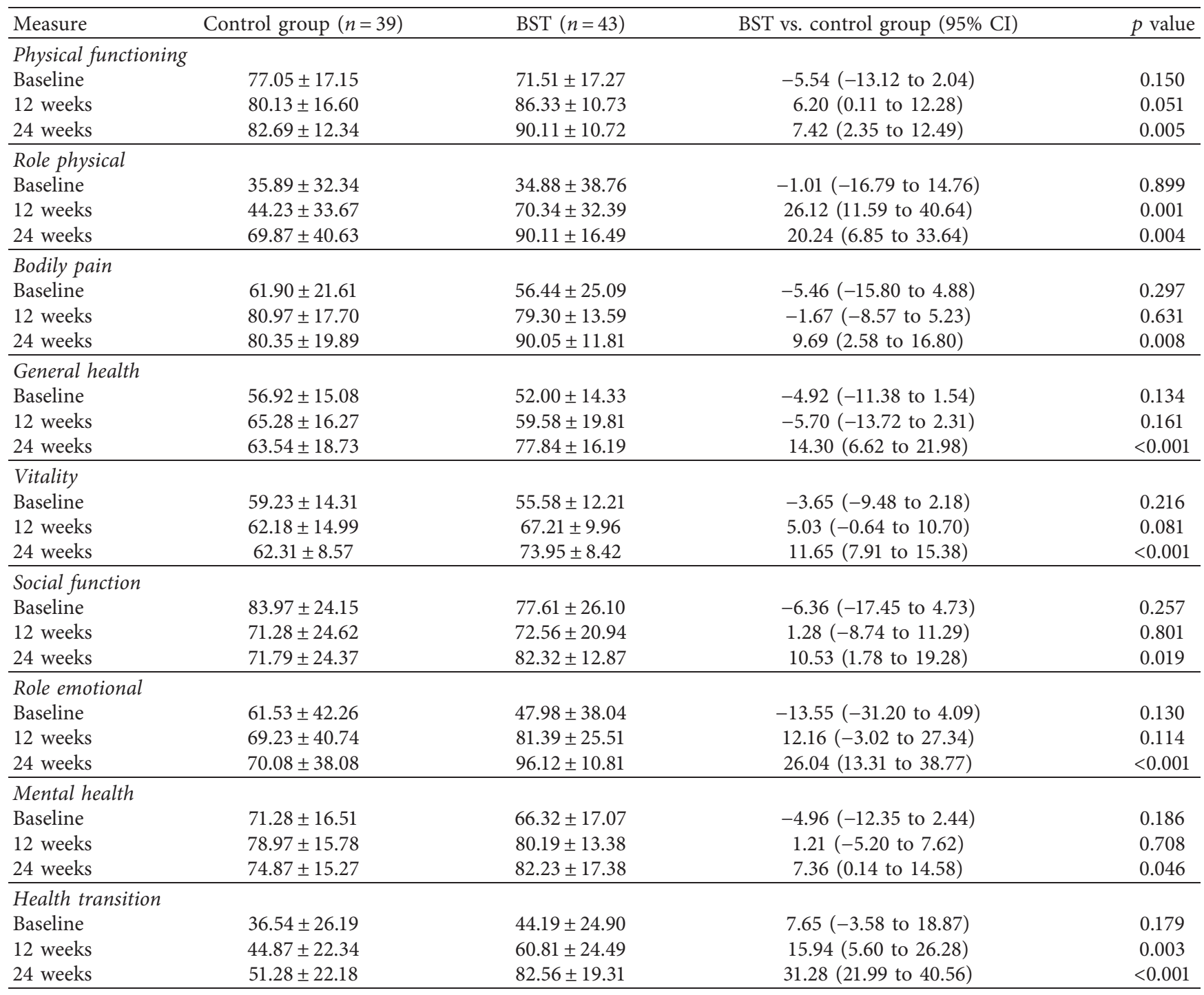

Data are presented as mean \pm SD. $p$ values were calculated by $t$-test for continuous variables.

TABLE 3: The changes of abdominal circumference and BMI during the 24 weeks.

\begin{tabular}{lcccc}
\hline Measure & Control group $(n=39)$ & BST $(n=43)$ & BST vs. control group (95\% CI) & $p$ value \\
\hline $\begin{array}{l}\text { Abdominal circumference } \\
\text { Baseline }\end{array}$ & $90.84 \pm 9.44$ & & & \\
12 weeks & $90.34 \pm 7.00$ & $87.87 \pm 6.68$ & $-2.96(-6.61$ to 0.68$)$ & 0.109 \\
24 weeks & $90.26 \pm 7.10$ & $86.89 \pm 5.25$ & $-4.29(-7.18$ to -1.39$)$ & 0.016 \\
\hline$B M I$ & & & & 0.004 \\
Baseline & $24.62 \pm 2.86$ & $23.95 \pm 2.35$ & $-0.67(-1.81$ to 0.48$)$ & 0.249 \\
12 weeks & $24.57 \pm 2.77$ & $23.94 \pm 2.33$ & $-0.63(-1.75$ to 0.49$)$ & 0.268 \\
24 weeks & $24.60 \pm 2.82$ & $23.40 \pm 2.30$ & $-1.21(-2.34$ to -0.09$)$ & 0.035 \\
\hline
\end{tabular}

BMI, body mass index. Data are presented as mean \pm SD.

cardiac function in patients with AMI after PCI. It could also significantly decrease BMI and abdominal circumference. No serious adverse events were observed during BST training, indicating the safety and usefulness of early intervention for patients with AMI who underwent PCI.
AMI is a common cardiac emergency with a high mortality rate [1]. Although PCI and drugs are currently effective in treating patients, many studies have shown that even after treatment, there is still a high risk of cardiovascular disease recurrence and death after PCI, especially 


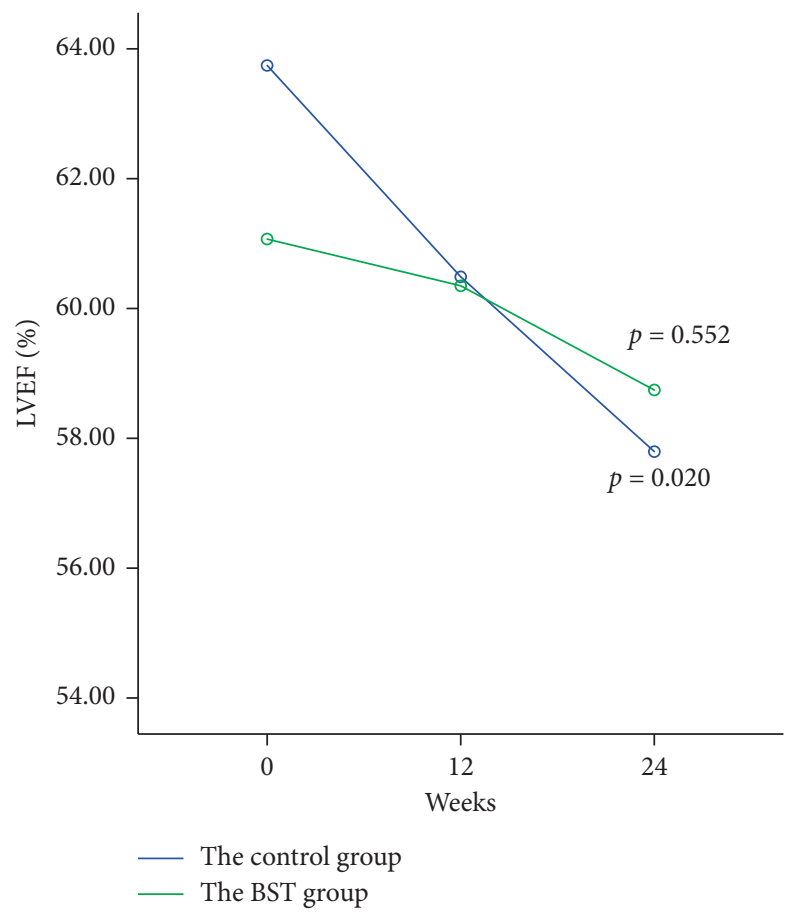

FIGURE 2: The changes of cardiac function during the 24 weeks. In the 24-week follow-up, the LVEF of the BST group and the control group was baseline (61.06 \pm 9.64 and $63.74 \pm 8.51$, respectively), 12-week outcome (60.34 \pm 9.20 and $60.48 \pm 9.64$, respectively), and 24 -week outcome (58.74 \pm 10.10 and $57.79 \pm 9.56$, respectively). A significant difference was found in the decline of the LVEF in the control group $(p=0.020)$. A nonsignificant difference was found in the decline of the LVEF in the BST group $(p=0.552)$.

within 1 year after surgery $[26,27]$. Therefore, there is considerable concern about this problem. Relevant literature reports that nearly $60 \%$ of patients after PCI are severely deficient in exercise, and about $84 \%$ of patients after PCI have associated anxiety symptoms, while $67 \%$ have associated depressive symptoms [26, 28]. Previous studies have shown that myocardial ischemia-reperfusion (IR) injury can cause ventricular cell death and is a major pathological event leading to morbidity and mortality in those with acute myocardial infarction $[11,12]$. Interestingly, appropriate exercise can rapidly produce a cardiac phenotype that resists IR-induced myocardial injury. It is essential for the prognosis of patients with myocardial infarction [13, 15]. Goel and his colleagues counted the proportion of patients with cardiac stents participating in cardiac rehabilitation in a state in the United States and found that poststent exercise rehabilitation had become a trend [8]. Exercise training is beneficial to the patient's cardiac rehabilitation.

To the best of our knowledge, our study is the first RCT to investigate the effect of BST exercise in patients with AMI who underwent PCI. Chen and his colleagues [29] have indicated that Baduanjin exercise can effectively improve the quality of life in patients with heart failure. A meta-analysis of nineteen studies [30] also showed that Baduanjin practice is beneficial for the quality of life. But the benefits of Baduanjin on the quality of life are more evident in older adults and individuals with chronic conditions. In our study, we were studying more severely ill patients with AMI and the results showed that the patients in the BST group performed significantly better than those in the control group at the 24- week outcome visit. Nine aspects of SF-36 were improved compared to the control group. These results were also similar to the findings of Tsang et al. [31].

As we have known, Baduanjin is a low-intensity, aerobic exercise and it is easy to learn because it is less demanding of physical and cognitive skills [32]. Several recent studies have demonstrated other benefits of Baduanjin exercise, including preventing ischaemic stroke, relieving pain and stiffness, and improving sleep quality and psychological well-being $[21,22,33]$. The BST consists of the sitting and the standing Baduanjin. It is developed by our team based on the characteristics of patients with myocardial infarction. The sitting Baduanjin exercise conforms to the aspects of lowintensity and long-term aerobic activity, which is very suitable for the rehabilitation training of patients with AMI after PCI. It was conducted twice a day in the hospital, for $30 \mathrm{~min} / \mathrm{session}$. The standing Baduanjin is more suitable for patients with sequential rehabilitation after discharge. Previous studies have shown that the reduction of LVEF following AMI is a result of infarcted myocardium and may involve dysfunctional but viable myocardium [20,34]. In our study, a nonsignificant difference was found in the decline of the LVEF in the BST group. This shows that BST exercise can prevent the decline of cardiac function. The BST also reduced $50 \mathrm{pg} / \mathrm{ml}$ on the NT-pro-BNP at 24 weeks, compared with the control group. Regarding BMI, there was no improvement between the two groups at 12 weeks. However, the participants in the BST group had a mean decrease of 1.21 in BMI compared with the control group at the 24 weeks, which was consistent with a study by An et al. 


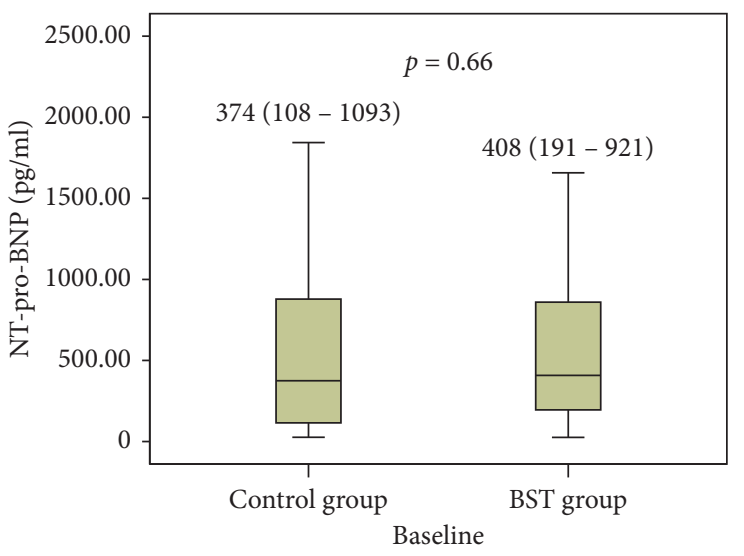

(a)

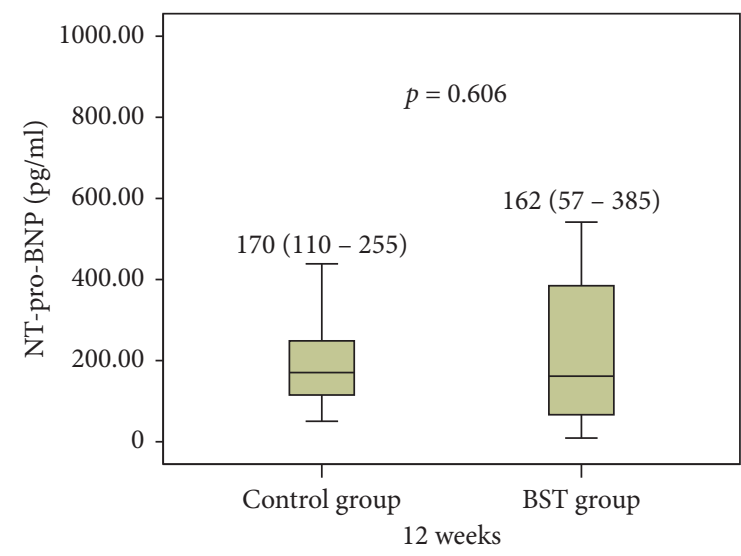

(b)

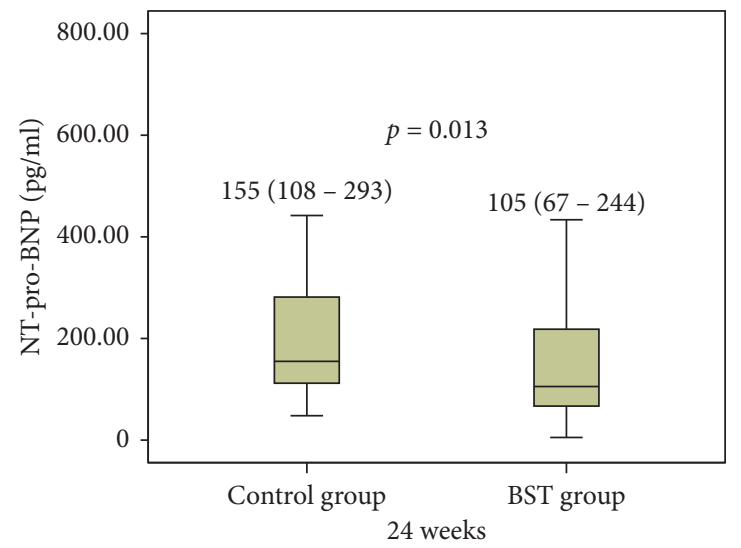

(c)

Figure 3: The changes of NT-pro-BNP during the 24 weeks. Values are presented as median with the interquartile range. A significant difference was found in the NT-pro-BNP between the BST and control groups at 24 weeks $(p<0.05)$; a nonsignificant difference was found between the BST and control groups at baseline and 12 weeks $(p>0.05)$.

[35], who reported that the BMI values of patients with knee osteoarthritis were significantly improved after one year of Baduanjin exercise. The BST group also had significantly better abdominal circumference after the 12 weeks and 24 weeks of intervention. The results of the analysis indicated that prolonged BST exercise was of benefit to the health of the patients with AMI who underwent PCI.

The performance of BST can systematically enhance cardiopulmonary functions and concurrently modulate mind and spirit, ultimately achieving the integration of the mind and body. However, the mechanisms behind the therapeutic change remain less understood and warrant future exploration.

4.1. Study Limitations. This study has some limitations. First, this is a single-center study. Second, because they were given behavior-based treatments, the participants were aware of their intervention assignments. Moreover, we are unable to provide a definitive physiological mechanism for the effects of the Baduanjin exercise. Nonetheless, this study offers informative data from the first large-scale clinical trial of BST in patients with AMI after PCI.

\section{Conclusions}

The BST appears to improve the quality of life in patients with AMI after PCI, with additional benefits of lowered abdominal circumference and BMI and improved level of cardiac function.

\section{Data Availability}

The data used to support the findings of this study are available from the corresponding author upon request.

\section{Disclosure}

This paper represents independent research. The views expressed are those of the author(s) and not necessarily those of the National Health Service, the NIHR, or the Department of Health and Social Care.

\section{Conflicts of Interest}

The author(s) declare no potential conflicts of interest for the research, authorship, and/or publication of this article. 


\section{Authors' Contributions}

Ming-Gui Chen and Xuefei Liang contributed equally to this work.

\section{Acknowledgments}

The authors gratefully acknowledge the contributions of all the investigators and participants of the carers. This work was funded by a grant (2014A020221092), from the Department of Science and Technology, Guangdong Provincial Academy of Medicine Joint Research Projects. This research was also financially supported by the Science Foundation of Guangdong Province (Nos. 2015A030310437, 2015A030306049, 2014A030313402, and 2014A020221048) and the Scientific and Technological Research of Traditional Chinese Medicine, Guangdong Provincial Hospital of Chinese Medicine (No. YN2016HL03).

\section{Supplementary Materials}

Supplementary Appendix Figure 1: (a) the introduction of the sitting Baduanjin exercises by the intensive care unit of Guangdong Provincial Hospital of Chinese Medicine. (b) the introduction of the standing Baduanjin exercises by the intensive care unit of Guangdong Provincial Hospital of Chinese Medicine. Supplementary Appendix Video 1: cardiac rehabilitation exercises of the Baduanjin sequential therapy. (Supplementary Materials)

\section{References}

[1] J. L. Anderson, E. W. Campion, and D. A. Morrow, "Acute myocardial infarction," New England Journal of Medicine, vol. 376, no. 21, pp. 2053-2064, 2017.

[2] F. Arslan, L. Bongartz, J. M. ten Berg et al., "2017 ESC guidelines for the management of acute myocardial infarction in patients presenting with ST-segment elevation: comments from the Dutch ACS working group," Netherlands Heart Journal, vol. 26, no. 9, pp. 417-421, 2018.

[3] P.-d. Liao, K.-j. Chen, J.-b. Ge, and M.-z. Zhang, "Clinical practice guideline of integrative Chinese and western medicine for acute myocardial infarction," Chinese Journal of Integrative Medicine, 2019.

[4] A. Moran, D. Gu, D. Zhao et al., "Future cardiovascular disease in China: markov model and risk factor scenario projections from the coronary heart disease policy modelChina," Circulation: Cardiovascular Quality and Outcomes, vol. 3, no. 3, pp. 243-252, 2010.

[5] N. Weiss-Faratci, I. Lurie, Y. Benyamini, G. Cohen, U. Goldbourt, and Y. Gerber, "Optimism during hospitalization for first acute myocardial infarction and long-term mortality risk: a prospective cohort study," Mayo Clinic Proceedings, vol. 92, no. 1, pp. 49-56, 2017.

[6] S. Chaudhury, R. Saini, A. K. Bakhla, and J. Singh, "Depression and anxiety following coronary artery bypass graft: current Indian scenario," Cardiology Research and Practice, vol. 2016, Article ID 2345184, 6 pages, 2016.

[7] J. S. M. Chan, R. T. H. Ho, K.-f. Chung et al., "Qigong exercise alleviates fatigue, anxiety, and depressive symptoms, improves sleep quality, and shortens sleep latency in persons with chronic fatigue syndrome-like illness," Evidence-Based
Complementary and Alternative Medicine, vol. 2014, Article ID 106048, 10 pages, 2014.

[8] K. Wang, R. J. Lennon, R. T. Tilbury, R. W. Squires, and R. J. Thomas, "Impact of cardiac rehabilitation on mortality and cardiovascular events after percutaneous coronary intervention in the community," Circulation, vol. 123, no. 21, pp. 2344-2352, 2011.

[9] O. J. Mechanic, S.A.G. Acute myocardial infarction, StatPearls Publishing LLC, Treasure Island, FL, USA, 2019.

[10] S. Johansson, A. Rosengren, K. Young, and E. Jennings, "Mortality and morbidity trends after the first year in survivors of acute myocardial infarction: a systematic review," BMC Cardiovascular Disorders, vol. 17, no. 1, 2017.

[11] S. Hashmi and S. Al-Salam, "Acute myocardial infarction and myocardial ischemia-reperfusion injury: a comparison," International Journal of Clinical and Experimental Pathology, vol. 8, no. 8, pp. 8786-8796, 2015.

[12] L. De Roeck, S. Vandamme, B. R. Everaert et al., “Adiponectin and ischemia-reperfusion injury in ST segment elevation myocardial infarction," European Heart Journal: Acute Cardiovasc Care, vol. 5, no. 1, pp. 71-76, 2016.

[13] S. K. Powers, A. J. Smuder, A. N. Kavazis, and J. C. Quindry, "Mechanisms of exercise-induced cardioprotection," Physiology, vol. 29, no. 1, pp. 27-38, 2014.

[14] R. Bolli and E. Marbán, "Molecular and cellular mechanisms of myocardial stunning," Physiological Reviews, vol. 79, no. 2, pp. 609-634, 1999.

[15] F. Besnier, M. Labrunée, A. Pathak et al., "Exercise traininginduced modification in autonomic nervous system: an update for cardiac patients," Annals of Physical and Rehabilitation Medicine, vol. 60, no. 1, pp. 27-35, 2016.

[16] H. Zhang and R. Chang, "Effects of exercise after percutaneous coronary intervention on cardiac function and cardiovascular adverse events in patients with coronary heart disease: systematic review and meta-analysis," Journal of Sports Science \& Medicine, vol. 18, no. 2, pp. 213-222, 2019.

[17] S. Yamamoto, K. Hotta, E. Ota, R. Mori, and A. Matsunaga, "Effects of resistance training on muscle strength, exercise capacity, and mobility in middle-aged and elderly patients with coronary artery disease: a meta-analysis," Journal of Cardiology, vol. 68, no. 2, pp. 125-134, 2016.

[18] F. Giallauria, W. Acampa, F. Ricc et al., "Exercise training early after acute myocardial infarction reduces stress-induced hypoperfusion and improves left ventricular function," $E \mathbf{E}$ ropean Journal of Nuclear Medicine and Molecular Imaging, vol. 40, pp. 315-324, 2013.

[19] T. C. A. Peixoto, I. Begot, D. W. Bolzan et al., "Early exercisebased rehabilitation improves health-related quality of life and functional capacity after acute myocardial infarction: a randomized controlled trial," Canadian Journal of Cardiology, vol. 31, no. 3, pp. 308-313, 2015.

[20] S. Mao, X. Zhang, B. Shao et al., "Baduanjin exercise prevents post-myocardial infarction left ventricular remodeling (BEPREMIER trial): design and rationale of a pragmatic randomized controlled trial," Cardiovascular Drugs and Therapy, vol. 30, no. 3, pp. 315-322, 2016.

[21] F. K. Cheng, "Effects of Baduanjin on mental health: a comprehensive review," Journal of Bodyweight and Movement Therapies, vol. 19, no. 1, pp. 138-149, 2015.

[22] M. Chen, H. Liu, H. Huang, and A. Chiou, "The effect of a simple traditional exercise programme (Baduanjin exercise) on sleep quality of older adults: a randomized controlled trial," International Journal of Nursing Studies, vol. 49, no. 3, pp. 265-273, 2012. 
[23] X. Lu, C. W. Hui-Chan, and W. W. Tsang, "Effects of Tai Chi training on arterial compliance and muscle strength in female seniors: a randomized clinical trial," European Journal of Preventive Cardiology, vol. 20, no. 2, pp. 238-245, 2013.

[24] D. M. Tehrani and A. H. Seto, "Third universal definition of myocardial infarction: update, caveats, differential diagnoses," Cleveland Clinic Journal of Medicine, vol. 80, no. 12, pp. 777-786, 2013.

[25] L. Li, H. M. Wang, and Y. Shen, "Chinese SF-36 health survey: translation, cultural adaptation, validation, and normalisation," Journal of Epidemiology and Community Health, vol. 57, no. 4, pp. 259-263, 2002.

[26] D. De Smedt, E. Clays, S. Höfer et al., "Validity and reliability of the HeartQoL questionnaire in a large sample of stable coronary patients: the EUROASPIRE IV study of the European Society of Cardiology," European Journal of Preventive Cardiology, vol. 23, no. 7, pp. 714-721, 2016.

[27] Y. Soga, H. Yokoi, K. Amemiya, M. Iwabuchi, and M. Nobuyoshi, "Safety and efficacy of exercise training after coronary stenting in patients with stable coronary artery disease," Circulation Journal, vol. 75, no. 10, pp. 2379-2386, 2011.

[28] R. Stewart, C. Held, R. Brown et al., "Physical activity in patients with stable coronary heart disease: an international perspective," European Heart Journal, vol. 34, pp. 3282-3293, 2013.

[29] D. M. Chen, W. C. Yu, H. F. Hung et al., "The effects of Baduanjin exercise on fatigue and quality of life in patients with heart failure: a randomized controlled trial," European Journal of Cardiovascular Nursing, vol. 17, no. 5, pp. 456-466, 2018.

[30] L. Zou, J. E. SasaKi, H. Wang et al., “A systematic review and meta-analysis baduanjin Qigong for health benefits: randomized controlled trials," Evidence Based Complementary and Alternative Medicine, vol. 2017, Article ID 4548706, 17 pages, 2017.

[31] H. W. Tsang, C. K. Mok, Y. T. A. Yeung, and S. Y. Chan, "The effect of Qigong on general and psychosocial health of elderly with chronic physical illnesses: a randomized clinical trial," International Journal of Geriatric Psychiatry, vol. 18, no. 5, pp. 441-449, 2003.

[32] P. R. Lawler, K. B. Filion, and M. J. Eisenberg, "Efficacy of exercise-based cardiac rehabilitation post-myocardial infarction: a systematic review and meta-analysis of randomized controlled trials," American Heart Journal, vol. 162, no. 4, pp. 571-584, 2011.

[33] G. Zheng, Q. Fang, B. Chen et al., "Qualitative evaluation of baduanjin (traditional Chinese Qigong) on health promotion among an elderly community population at risk for ischemic stroke," Evidence Based Complementary and Alternative Medicine, vol. 2015, Article ID 893215, 10 pages, 2015.

[34] G. Ndrepepa, S. Cassese, M. Emmera et al., "Relation of ratio of left ventricular ejection fraction to left ventricular enddiastolic pressure to long-term prognosis after ST-segment elevation acute myocardial infarction," The American Journal of Cardiology, vol. 123, no. 2, pp. 200-205, 2019.

[35] B. C. An, Y. Wang, X. Jiang et al., "Effects of Baduanjin exercise on knee osteoarthritis: a one-year study," Chinese Journal of Integrative Medicine, vol. 19, no. 2, pp. 143-148, 2013. 